\section{Nanoscale resolution in GFP-based microscopy}

\author{
Katrin I Willig, Robert R Kellner, Rebecca Medda, \\ Birka Hein, Stefan Jakobs \& Stefan W Hell \\ We report attainment of subdiffraction resolution using \\ stimulated emission depletion (STED) microscopy with GFP- \\ labeled samples. The $\sim 70 \mathrm{~nm}$ lateral resolution attained in this \\ study is demonstrated by imaging GFP-labeled viruses and the \\ endoplasmic reticulum (ER) of a mammalian cell. Our results \\ mark the advent of nanoscale biological microscopy with \\ genetically encoded markers.
}

The resolution of a light-focusing microscope is usually limited to $\Delta r=\lambda / 2 \mathrm{NA} \approx 200 \mathrm{~nm}$, with NA denoting the numerical aperture of the lens and $\lambda$ the wavelength of light ${ }^{1}$. Because fluorescence microscopy is the most popular imaging mode for subcellular research, the diffraction resolution limit severely hampers life science studies. Image deconvolution may increase the resolution by up to a factor of two, but further improvement using mathematics is viable only by using a priori knowledge of the sample ${ }^{2}$.

STED microscopy ${ }^{3,4}$ is distinct from commonly applied fluorescence microscopy modes because its resolution is no longer strictly limited by $\lambda$. Rather it follows that:

$$
\Delta r \cong \lambda /(2 \mathrm{NA} \sqrt{1+\zeta})
$$

with $\zeta$ describing the magnitude of STED ${ }^{5,6}$. Hence, the maximum resolution attainable using this microscope is given by the largest practical value of $\zeta$, whereas the conceptual limit is about the size of the label.

STED microscopy has proven to be decisive in solving key problems in biology. For example, it was used to establish that the synaptic vesicle protein synaptotagmin I forms clusters on the

Figure 1 | STED microscope for GFP imaging. (a) Blue (490 $\mathrm{nm}$ ) excitation light forms a regular Airy disk in the lens focal plane and is overlapped with a yellow $(575 \mathrm{~nm})$ doughnut spot for STED. The doughnut is produced by applying a helical phase delay on the STED beam wavefront, $\exp (i \phi)$, with $0 \leq \phi \leq 2 \pi$, using a programmable wavefront modulator (PAL-SLM, Hamamatsu Photonics). The linear helical progression of the phase from 0 to $2 \pi$ ensures that the light field from opposite parts of the entrance pupil of the lens interferes destructively on the optic axis. The focal intensity thus assumes a cylindrical shape centered around the dark optic axis. (b,c) Excitation, fluorescence and stimulated emission in the energy diagram (b) in relation to the excitation and emission spectrum of GFP (c). STED is performed at the red tail of GFP emission. presynaptic membrane ${ }^{7}$, a fact which has crucial implications for synaptic protein recycling. It also revealed that the Drosophila sp. protein bruchpilot forms ring-like structures that define the synaptic active zones in the Drosophila sp. neuromuscular junction ${ }^{8}$. Furthermore, it proved that the SNARE protein syntaxin remains aggregated in the plasma membrane even upon massive overexpression ${ }^{9}$. All imaging applications using STED so far have relied on organic dyes that stained organelles either directly ${ }^{4}$ or through antibody labeling ${ }^{10}$. Here we reveal that nanoscale resolution can also be realized with fluorescent proteins.

A STED microscope design capable of imaging GFP is shown in Figure 1 (see Supplementary Methods online for details). The protein is excited at $\lambda=490 \mathrm{~nm}$ using a laser diode emitting 100-ps pulses (PicoTA, Picoquant) operating at $80 \mathrm{MHz}$. The resulting focal excitation spot with a full-width-half-maximum (FWHM) of $240 \mathrm{~nm}$ represents the resolution obtained by conventional microscopy, that is, the Airy disk of the 1.4 NA lens used (Planapo oil, $100 \times$; Leica).

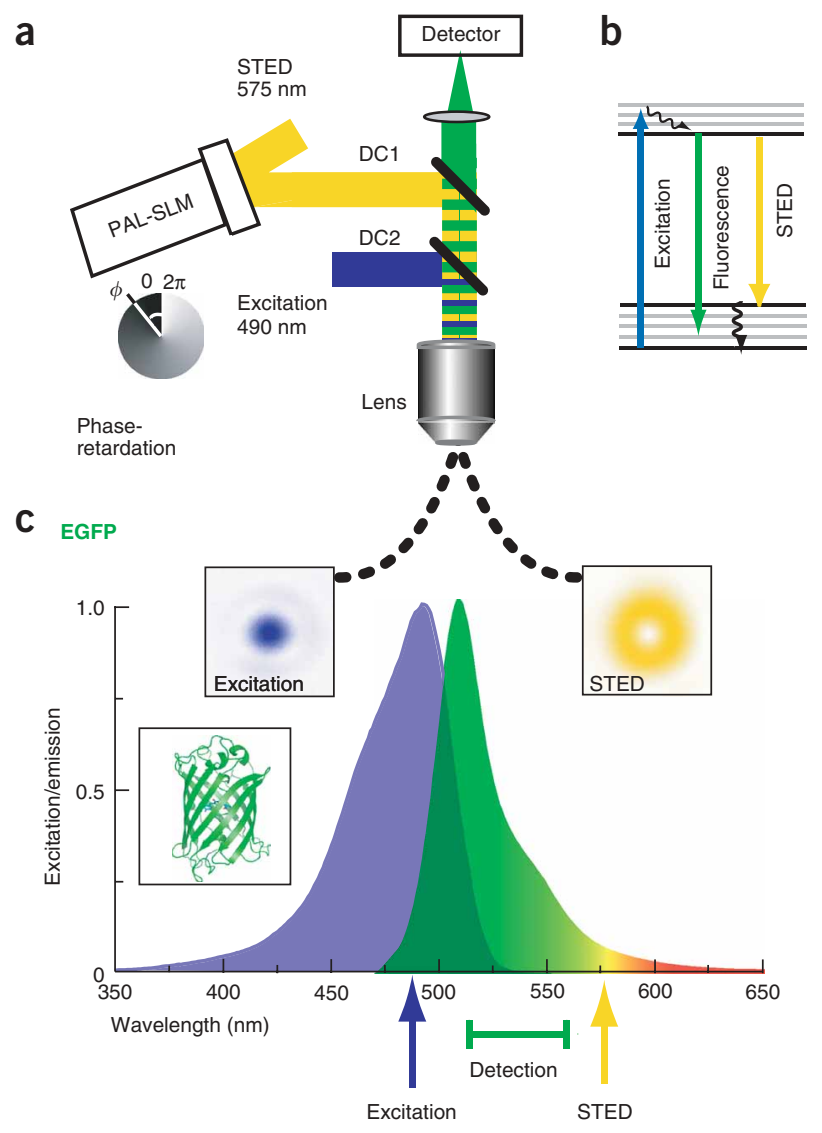

Max Planck Institute for Biophysical Chemistry, Department of NanoBiophotonics, Am Fassberg 11, 37077 Göttingen, Germany. Correspondence should be addressed to S.W.H. (shell@gwdg.de).

RECEIVED 5 JUNE; ACCEPTED 17 JULY; PUBLISHED ONLINE 9 AUGUST 2006; DOI:10.1038/NMETH922 


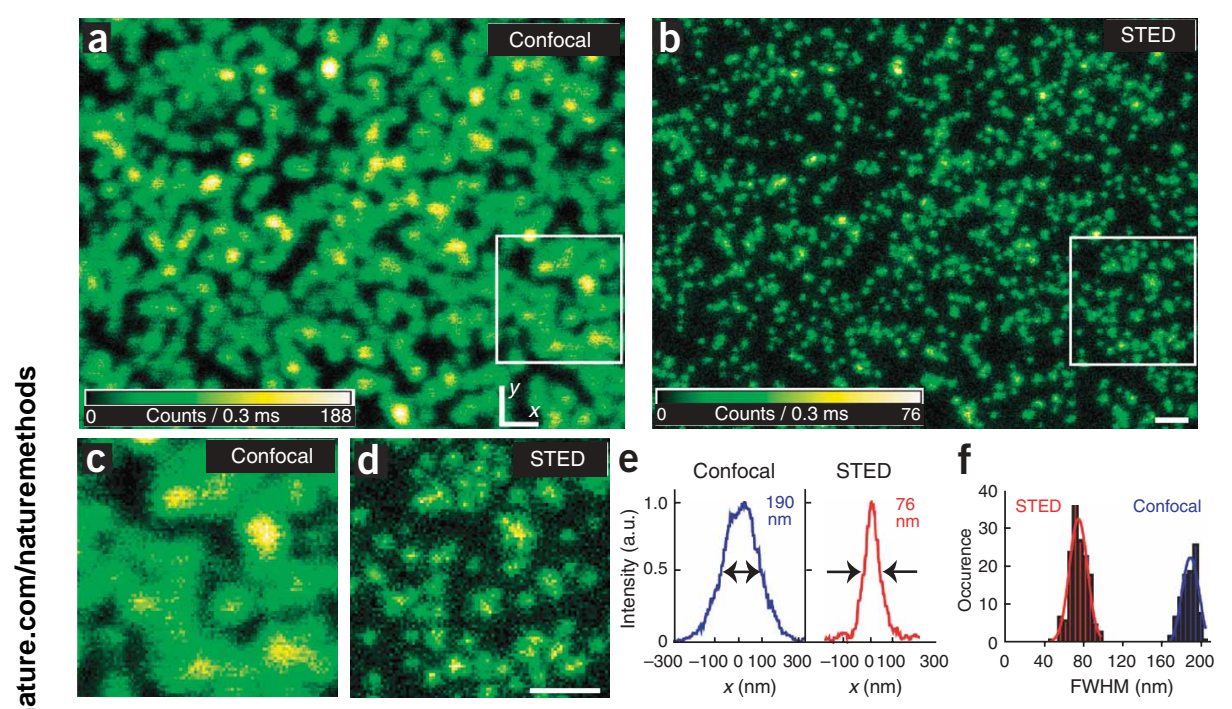

Figure 2 | STED overcomes the diffraction resolution barrier in GFP-based microscopy. $(\mathbf{a}, \mathbf{b})$ GFP-labeled rotavirus-derived particles imaged confocally (a) and with STED (b). The threefold increase in lateral resolution in $\mathbf{b}$ separates many more of the $40-\mathrm{nm}$ sized particles. (c,d) Magnified view of the marked area demonstrates blurred blobs in the confocal image (c), whereas most of the particles are resolved by STED (d). (e) Raw data profile through an overlay of ten individual particles displays a FWHM of 190 $\mathrm{nm}$ for the confocal and $76 \mathrm{~nm}$ for STED mode. (f) Histograms of the FWHM detail the decrease of spot size. Scale bars, $500 \mathrm{~nm}$.

To overcome this limit, the excitation spot is overlaid with a doughnut-shaped counterpart, the STED beam, which provides 200-ps pulses of lower-energy photons for molecular de-excitation, synchronized at $80 \mathrm{MHz}$. The fluorescent state depopulates as $\exp (-\zeta) \equiv \exp (-\sigma \Phi)$, with $\Phi$ denoting the number of stimulating photons per pulse and per area. With a typical cross-section for stimulated emission, $\sigma \approx 10^{-17} \mathrm{~cm}^{2}$, we require $\Phi>10^{17}$ photons $/ \mathrm{cm}^{2}$ to obtain $\zeta>1$ and hence subdiffraction values $\Delta r$. As the doughnut area is $\sim 10^{-9} \mathrm{~cm}^{2}$, the STED pulse should contain at least $10^{8}$ photons, corresponding to a STED beam average power on the order of $P \approx 3 \mathrm{~mW}$.

The value of $P$ to be actually applied depends inversely on the precise value of $\sigma$, which in turn scales with the emission spectrum of the dye ${ }^{11}$. Tuning the STED pulse to the peak emission $(510 \mathrm{~nm})$, however, is not advisable for imaging GFP, because of the residual excitation at this wavelength (Fig. 1c). Screening revealed that for imaging GFP, STED prevails from 570 to $600 \mathrm{~nm}$. Therefore, we optimized our emission filter to detect fluorescence in the 510-560 $\mathrm{nm}$ range. We focused the fluorescence onto a detector opening that was equivalent to common pinhole settings used in confocal microscopy ${ }^{1}$. Confocalization is not required, but it conveniently provides confocal axial resolution and, by blocking the STED beam, direct comparison with this standard technique. We acquired the images by scanning the sample with a piezo stage.

Figure 3 | Subdiffraction imaging of GFP-labeled ER in PtK2 cell (a-d) Confocal (a) and STED (b) images. The improved resolution in $\mathbf{b}$ is also evident in $\mathbf{c}$ and $\mathbf{d}$ displaying magnified views of the boxed regions in $\mathbf{a}$ and $\mathbf{b}$, respectively; data is smoothed by a Gaussian filter over 1.5 pixels. $(\mathbf{e}, \mathbf{f})$ Images after a regularized linear deconvolution using the PSF obtained from Figure 2. The arrows indicate details in deconvolved STED data (f) that are not obtainable via deconvolution of confocal data (e). Scale bars, $1 \mu \mathrm{m}$.
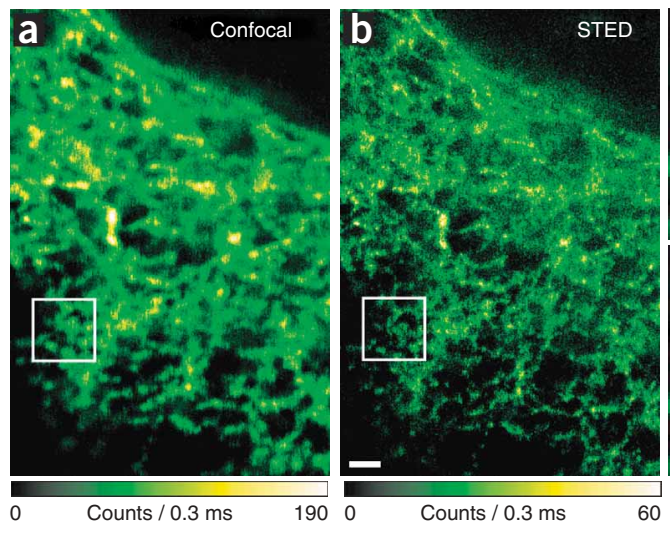

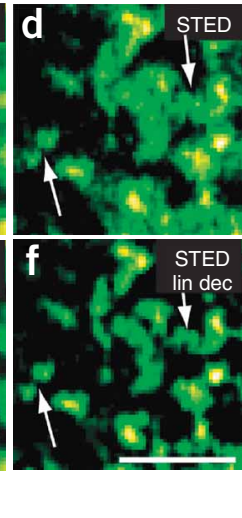

To explore the resolution, we tuned to $575 \mathrm{~nm}$ and imaged rotavirus-derived particles $^{12}$. The rotavirus is trilayered, containing the VP2 proteins in the innermost layer, VP6 in the medial layer, and VP4 and VP7 in its outermost coat. Coexpression of VP6 and GFP-tagged VP2 in a baculovirus expression system yields double-layered, GFP-tagged virus-like particles containing GFP-VP2 molecules in their 40-nm diameter inner capsids ${ }^{12}$.

Comparison of confocal and STED recording at $7.2 \mathrm{~mW}$ (excitation power $70 \mathrm{nW}$ ) reveals the improved resolution of the latter technique (Fig. 2). The virus-like particles are individually resolved in areas where confocal microscopy only renders blurred patches, and this finding is substantiated by the raw data profiles (Fig. 2e). Featuring an FWHM of $190 \mathrm{~nm}$, the confocal profile is basically the point-spread function (PSF) of a confocal microscope ${ }^{1}$. The profile in the STED images displays an FWHM of $76 \mathrm{~nm}$. Considering the $40-\mathrm{nm}$ capsid, it follows that the PSF of the STED imaging mode features an FWHM of $\sim 70 \mathrm{~nm}$.

To label the ER of fixed mammalian cells, we expressed GFP fused to an ER-targeting sequence in rat kangaroo kidney epithelial (PtK2) cells (Fig. 3). Comparison between the confocal and the STED recordings reveals the improved resolution of the latter. Linear deconvolution of raw data increases the resolution further.

The addition of the STED doughnut reduced the spot area by ninefold, but, owing to its cylindrical shape, did not squeeze the spot axially. Therefore, in a convoluted organelle like the ER, the improved lateral resolution alone cannot unfold the method's full power. Remedies to this problem that leave the cell intact are an 
additional STED beam with specific peaks above and below the focal plane, or the combination of STED with 4 Pi microscopy ${ }^{10}$.

Keeping the intensity at the doughnut center close to zero is imperative in STED microscopy, because a finite STED beam intensity at the minimum reduces the flux of the fluorescence photons from the central spot to be recorded. If the central minimum amounts to $b$ percent of the doughnut crest, the peak flux is approximately $\exp (-0.01 b \zeta)$ times its value without STED, meaning that $b$ should not exceed $\sim 5 \%$. Therefore, the application क of STED microscopy in scattering media, such as GFP-labeled tissue, depends on how well the 'zero' can be kept free of scattered STED light. Our preliminary results with organic dyes display subdiffraction resolution several cell layers deep. Several options can readily be envisaged to keep $b$ low, such as the application of active optical elements and of annihilating light fields.

We are confident that the resolution can be further augmented by refining the pulse duration and the timing. Live-cell GFP imaging should be facilitated by implementing rapid image acquisition through beam scanning. The most straightforward option is to merge the excitation and the phase-modified STED beam in a common path and subject both beams to fast galvanometer scanning. As any increase in resolution calls for finer image pixelation, it will be preferable to reduce the field of view by the same factor by which the spot area has been decreased. Because the spot size can be tuned by adjusting $\zeta$, one can balance between the degree of resolution on the one hand, and the signal strength, the imaging speed and the field of view on the other hand. In the future, this zooming should be possible while scanning.

We also obtained $\sim 70-\mathrm{nm}$ resolution with the enhanced yellow fluorescent protein (EYFP) by applying STED at $595 \mathrm{~nm}$. The yellow protein $m$ Citrine ${ }^{13}$ can also be depleted at this wavelength. The red fluorescent protein $\mathrm{mRFP}^{14}$ exhibits STED at $710 \mathrm{~nm}$. Screening of recent additions to the fluorescent protein family ${ }^{15}$ is unraveling further options; for example, mStrawberry lends itself to depletion at $666 \mathrm{~nm}$.

De-excitation by stimulated emission is as fundamental a molecular process as excitation. STED is therefore prevalent in all fluorophores, reaching its maximum efficiency at predefined wavelengths and pulse durations. In fact, screening of organic dyes has so far shown that STED can be applied to any dye with a fluorescent lifetime $>0.8 \mathrm{~ns}$. Therefore, STED microscopy is compatible with multicolor labeling. A possible combination of functionalized organic dyes is Atto532 (AttoTech), Dy-510XL (Dyomics) and Atto647N (AttoTech), featuring green $(550 \mathrm{~nm})$, yellow $(590 \mathrm{~nm})$ and red $(670 \mathrm{~nm})$ emission peaks, respectively. We also expect fluorescent protein-based multicolor STED microscopy to be viable, for example with the set of proteins listed above. For several dye pairs, for example GFP and mCitrine-EYFP, the same excitation or STED wavelength can be applied, so that the set of required wavelengths can be conveniently reduced. Notwithstanding this, multicolor STED microscopy should be greatly facilitated by emerging technologies. Compact 'white light' sources such as diode-pumped photonic crystal fibers provide spatially clean whole-spectrum (500-900 nm) subnanosecond pulses that should be ideal for STED.

In conclusion, nanoscale fluorescent protein microscopy should open up a new avenue for answering many key questions in biology.

Note: Supplementary information is available on the Nature Methods website.

\section{ACKNOWLEDGMENTS}

Purified GFP-VLPs were provided by J. Cohen and the plasmid eGFP-ER by P. Lipp. We thank S. Verrier, T. Rosenmund, A.C. Schauss, J.J. Sieber and T. Müller for providing samples, A. Schönle for help with the software ImSpector, V. Westphal and B. Harke for valuable discussions, as well as J. Keller and B. Rankin for critical reading. We thank R.Y. Tsien for providing the plasmids coding for mCitrine, mStrawbery and mRFP.

\section{COMPETING INTERESTS STATEMENT}

The authors declare that they have no competing financial interests.

Published online at http://www.nature.com/naturemethods/ Reprints and permissions information is available online at http://npg.nature.com/reprintsandpermissions/

1. Pawley, J.B. (ed.) Handbook of biological confocal microscopy (Springer, New York, 2006).

2. Conchello, J-A. \& Lichtman, J.W. Nat. Methods 2, 920-931 (2005).

3. Hell, S.W. \& Wichmann, J. Opt. Lett. 19, 780-782 (1994).

4. Klar, T.A., Jakobs, S., Dyba, M., Egner, A. \& Hell, S.W. Proc. Natl. Acad. Sci. USA 97, 8206-8210 (2000).

5. Hell, S.W. Nat. Biotechnol. 21, 1347-1355 (2003).

6. Westphal, V. \& Hell, S.W. Phys. Rev. Lett. 94, 143903 (2005).

7. Willig, K.I., Rizzoli, S.O., Westphal, V., Jahn, R. \& Hell, S.W. Nature 440, 935-939 (2006).

8. Kittel, R.J. et al. Science 312, 1051-1054 (2006).

9. Sieber, J.J., Willig, K.I., Heintzmann, R., Hell, S.W. \& Lang, T. Biophys. J. 90, 2843-2851 (2006).

10. Dyba, M., Jakobs, S. \& Hell, S.W. Nat. Biotechnol. 21, 1303-1304 (2003).

11. Schäfer, F.P. Dye Lasers (Springer, Berlin, 1973).

12. Charpilienne, A. J. Biol. Chem. 276, 29361-29367 (2001).

13. Griesbeck, O., Baird, G.S., Campbell, R.E., Zacharias, D.A. \& Tsien, R.Y. J. Biol. Chem. 276, 29188-29194 (2001).

14. Campbell, R.E. et al. Proc. Natl. Acad. Sci. USA 99, 7877-7882 (2002).

15. Shaner, N.C., Steinbach, P.A. \& Tsien, R.Y. Nat. Methods 2, 905-909 (2005). 